\title{
El mundo que se hizo visible a través de lo afectivo* 1
}

\section{In The World That Affect Proposed}

0 mundo que se tornou visível através do afetivo 


\section{Kathleen C. Stewart**}

Professor, Department of Anthropology, University of Texas Austin.

Correo electrónico: kstewart@austin.utexas.edu

\section{Alejandro Ponce de León-Calero*** [Translator]}

Ph.D. Candidate ABD, Graduate Group Cultural Studies, University of California Davis

Correo electrónico: poncedeleon@ucdavis.edu

Revista Corpo-grafías: Estudios críticos de y desde los cuerpos / Volumen 6 - Número 6 / Enero - diciembre de 2019 / ISSN impreso 2390-0288, ISSN digital 2590-9398 / Bogotá, D.C., Colombia / 167-175.

Fecha de recepción: 8 de junio de 2018

Fecha de aceptación: 26 de agosto de 2018

Doi: https://doi.org/10.14483/25909398.14236

Cómo citar este artículo: Stewart, K. (2019, enero-diciembre). El mundo que se hizo visible a través de lo afectivo. Revista Corpo-grafías: Estudios críticos de y desde los cuerpos, 6(6), 167-175 / ISSN 2390-0288.

*Artículo de investigación: Affect came into view through a slowed ethnographic practice attuned to the forms and forces unfolding in scenes and encounters. In the world affect brought into view, the point of analysis was not to track the predetermined effects of abstractable logics and structures but, rather, to compose a register of the lived affects of the things that took place in a social-aesthetic-material-political worlding.

Para mí, lo afectivo apareció a través de una práctica etnográfica detenida, atenta, y en sintonía con las formas y fuerzas que se desarrollan en las escenas y en los encuentros. En el mundo de lo afectivo, el punto de análisis ya no era rastrear los efectos predeterminados de las lógicas y estructuras abstraíbles sino, más bien, componer un registro vivencial en donde ocurren cosas en relación al mundo material, social, estético, y político.

**Professor in the Department of Anthropology at the University of Texas, Austin, she writes and teaches on affect, the ordinary, the senses, and modes of ethnographic engagement based on curiosity and attachment. Her first book, A Space on the Side of the Road: Cultural Poetics in an 'Other' America (Princeton University Press, 1996) portrays a dense and textured layering of sense and form laid down in social use. Ordinary Affects (Duke University Press, 2007) maps the force, or affects, of encounters, desires, bodily states, dream worlds, and modes of attention and distraction in the composition and suffering of present moments lived as immanent events. Her current project, Worlding, tries to approach ways of collective living through or sensing out. An attunement that is also a worlding.

***An ethnographer and Ph.D. Srtudent in Cultural Studies at the University of California, Davis. He holds a BA in Political Science and a MA in Cultural studies from Universidad de los Andes (Bogotá, Colombia), as well as a MA in Sociology from the University of Texas, Austin. His work is concerned with the entanglements between armed violence and everyday life in impoverished and marginalized communities in Colombia, were armed confrontation has been routinized across various domains of both public and private life. His most recent research examines rebel and paramilitary violence against Colombian cattle ranchers in order to explore individual violent interactions as a way to understand the patterns for differentiated use of violence in war zones.

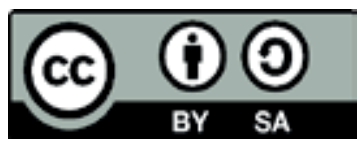




\section{Resumen}

Los estudios sobre los afectos y lo afectivo (Affect studies) hacen parte de una reciente búsqueda etnográfica por encontrar nuevas formas de describir lo que precede y excede en aquellas categorías con que definimos las labores de la vida de nuestro presente histórico. Para mí, lo afectivo apareció a través de una práctica etnográfica detenida, atenta, y en sintonía con las formas y fuerzas que se desarrollan en las escenas y en los encuentros. En el mundo de lo afectivo, el punto de análisis ya no era rastrear los efectos predeterminados de las lógicas y estructuras abstraíbles sino, más bien, componer un registro vivencial en donde ocurren cosas en relación al mundo material, social, estético, y político. Asimismo, en el mundo propuesto por lo afectivo, las cosas se empezaron a juntar, quizás con mucha fuerza, para hacerse reconocibles como algo en lo que se está, o se acerca, que opone y se resiste, pero que siempre es una composición aproximada de lo que ha sucedido. La escritura etnográfica también comenzó a narrar estados colectivos y sensibilidades que afectaban a las personas y que atravesaban cosas que, de otro modo, serían inconmensurables; haciendo que el aparato de la conceptualización se alinease con las preocupaciones inmanentes de dicha etnografía detenida y con las preocupaciones de los mundos que yo intentaba rastrear.

Palabras clave: afecto; embodiment; método etnográfico. 


\begin{abstract}
Affect studies is part of a renewed search for modes of ethnographic theory and critique that looks for ways to describe what precedes and exceeds the categorical in the labors of living through historical presents. For me, affect came into view through a slowed ethnographic practice attuned to the forms and forces unfolding in scenes and encounters. In the world affect brought into view, the point of analysis was not to track the predetermined effects of abstractable logics and structures but, rather, to compose a register of the lived affects of the things that took place in a social-aesthetic-materialpolitical worlding. In the world that affect proposed, things threw together, perhaps with a great deal of force, to become recognizable as something to be in, or near, or to oppose and resist, but it was always still an approximate composition of what was happening. Ethnographic writing began, again, to try to describe collective states and sensibilities hitting people and traversing otherwise incommensurate things, pulling the apparatus of conceptualization itself into a tricky alignment with slow ethnography's immanent concerns and with the concerns of the worlds it was trying to trace.
\end{abstract}

\title{
Keywords: affect; embodiment; ethnography.
}

\section{Resumo}

Estudos sobre os afetos e afetivo (Affect studies) fazem parte de uma recente missão etnográfica para encontrar novas maneiras de descrever o que precede e excede as categorias que definem o trabalho da vida de nosso presente histórico. Para mim, o afetivo apareceu através de uma prática etnográfica cuidadosa, atenta, e em linha com as formas e as forças de desenvolvimento nas cenas e encontros. No mundo do afetivo, o ponto da análise não era rastrear os efeitos padrão de estruturas lógicas e abstraível mas sim compor um registro experimental onde as coisas acontecem em relação ao material, social, estético e mundo político. Também no mundo afetivo proposta, as coisas começaram a se reunir, talvez muito difícil, para ser reconhecido como algo que é ou abordagens que se opõe e resiste, mas é sempre uma composição aproximada do que aconteceu. A escrita etnográfica também começou a narrar estados coletivos e sensibilidades que afetavam as pessoas e passavam por coisas que de outro modo seriam incomensuráveis; fazendo o aparato de conceitualização alinhar-se com as preocupações imanentes de tal etnografia presa e com as preocupações dos mundos que eu estava tentando rastrear.

\section{Palavras-chave: afeto; embodiment; método etnográfico.}


Para mí, lo afectivo se hizo visible a través de una práctica etnográfica lenta, en sintonía con las formas y con las fuerzas que se despliegan en ciertas escenas y encuentros. Esta práctica haló el andamiaje conceptual e hizo que entrara en una engorrosa alianza con las preocupaciones inmanentes a este tipo de ritmo etnográfico, así como con las preocupaciones propias a los mundos que intentaba rastrear. Los objetos antropológicos se convirtieron en cosas que resplandecían desde su punto de amalgamiento o que yacían de modo nasciente en la atmosfera. Estos objetos debían ser puestos en marcha, ser abordados desde ciertos ángulos, y ser vistos como estados del ser; emergían, o se encontraban suspendidos en potencialidad, o colapsaban, o eran residuos, o yacían en la materia viva como si esta fuera un lugar de reposo. Así, lo cultural se convirtió en un campo magnetizado y resonante, inscrito en las personas y en las cosas que conviven a través de eventualidades y condiciones. La escritura etnográfica comenzó, nuevamente, a tratar de describir los estados colectivos así como las sensibilidades que sacudían a las personas y que atravesaban cosas que de otra forma serían inconmensurables: modos de pensar, ensamblajes de infraestructuras e instituciones, nuevas ecologías, ritmos de la vida cotidiana y aquel extraño tejido que producen los dispositivos móviles y las redes sociales. En el mundo que se hizo visible a través de lo afectivo, el punto del análisis ya no era el de rastrear los efectos predeterminados por las lógicas y estructuras definidas a través de la abstracción, sino el de componer un registro de los afectos vividos que tuvieron lugar en un mudanear social-estético-material-político.

Todo esto significaba que, no sólo los sistemas y los modos del ser debían de ser vistos de manera más desordenada de lo que habrían permitido las teorías y modelos anteriores, sino que, además, las singularidades que circulaban en lo cotidiano, lo social, lo material, la infraestructura, la atmósfera, lo político y la ensoñación tomaran forma a través de una serie de precisiones. Lejos de ser concreciones o incorporaciones de alguna generalidad, estas singularidades emergían como los eventos de un mundo sociocultural en particular. Las singularidades daban vida a un campo energético constituido por los cambiantes afanes cotidianos. Estas brotaban de las problemáticas vividas en un presente y, por lo tanto, podían ser instrumentalizadas, desplegadas, maniobradas, sufridas, o ser utilizadas de manera lúdica. La socialidad, en sí misma, era un ritmo que le marcaba el paso a una saturación en un mundo. El sujeto y su agencia fueron sumergidos en el funcionamiento siempre fundamental, práctico y artístico de la diferencia y la expresividad; aquel que tiene lugar tanto en los grandes como en los pequeños eventos de lo cotidiano. El peso del mundo fue puesto sobre los hombros del sujeto y su agencia, y estos, a su vez, fueron cargados con intensidad, fracturados, y divididos en capas.

Por lo tanto, los afectos no eran simplemente el efecto secundario de los sistemas, los códigos, o los imaginarios localizados en otro lugar, sino, más bien, el registro de la vida misma como un ensamblaje de elementos que se 
ponen en contacto estético (ver Pine, 2012) entre sí durante el transcurso de los eventos. La dimensión de lo afectivo ayudó a que la antropología regresara nuevamente a los sentidos y a las sensaciones, a la materialidad, y a lo visceral. Proponía un mundo el cual es vivido pero que no está sencillamente anclado a la conciencia del sujeto humanista o a sus categorías de pensamiento. Por el contrario, un mundo cargado de afecto es una prolífica zona de contacto, de uso mixto, en estado de transición constante, que le permite a las personas "improvisar con lo ya sentido" (Manning, 2009, p. 30). Aquí, el sujeto emerge en la arriesgada labor de ser-en-el-mundo, a través de las diferentes formas que toman las potencias de lo cotidiano en el curso de la vida. La gente trabaja en la pura precariedad de las situaciones, entre la abrumadora avalancha de lo tendencial, de aperturas y clausuras, disoluciones y solidificaciones, como también en las cohesiones del entorno social, de un proyecto específico, o de un hábito arraigado. El sujeto afectivo es una persona que espera, en compañía de otros, a que las cosas lleguen. Es alguien que aprende a percibir lo que viene y la forma que esto podría tomar, alguien que nota qué se cristaliza y cómo las cosas rebotan y se recuperan en una ecología estética socio-natural de composiciones y límites de expresividad. Para el sujeto afectivo, el peso del mundo se realiza en lo que puede ser deseado y en lo que es temido, en lo que florece y en lo que le atañe. La vida es el experimento de ser-en-el mundo, de encontrar maneras de hacer parte de un circuito de fuerzas y formas. Es una aspiración a sacar algo de las transmutaciones alquímicas (Pine, 2016) de las cosas que, dentro de su propia trayectoria, se complican mucho más de lo que permiten los modelos humanistas sobre el sufrimiento o los registros híper-legibles de la normatividad y el Estado. La vida toma lugar en los gestos inhumanos de los demonios y de los ángeles, en el conflicto interno de los adictos y en la ira de los racistas, en la capacidad de resistir de quienes han sido heridos en modos increíbles o de los que, extrañamente, aún siguen curiosos.

El mundo al que hago referencia no está predispuesto sobre la mesa -como si la única tarea pendiente fuese aquella de evaluarlo. El giro afectivo complicó los viejos mantras que articulaban nuestra comprensión sobre la estructura, la mediación, la representación y la codificación, los cuales en su momento habían logrado operar como atajos a la cultura y al poder. Más allá de la simple crítica a la representación, lo afectivo trajo consigo una crítica que se ejerce en modo afirmativo pues explora, con sorpresa, qué y cómo ocurren las cosas. La labor aquí es la de esperar y así ver cómo se desenvuelven las cosas en un momento, tomar nota de los puntos de contacto, reconocer el peso o el olor de la atmósfera, o rastrear la distribución de las intensidades en los sujetos, objetos, instituciones, leyes, materialidades y especies. Lo cotidiano no es naturalizado como aquello que "aún no ha sido pensado", sino que es abordado cómo la generación prolífica de soluciones insólitas a problemáticas que, a su vez, han desencadenado situaciones inesperadas. Este es un mundo des-literalizado, en el cual las palabras y las cosas son polívocas; es uno en el cual no existe una división endémica entre un orden naturalizado dado y el excepcionalísimo propio al evento o a la movilización. 
En el mundo de lo afectivo, las cosas acaecen tal vez con demasiada fuerza, para hacerse reconocibles como algo en lo que se puede habitar, o estar cerca de, u oponerse y resistir, pero siempre como una composición que sólo se aproxima a lo que estaba sucediendo, un ángulo agudo, una impresión que rebota mientras se mira de reojo a lo que no fue capturado o reconocido. Una exposición de lo real como una alternancia rítmica que resplandece y se atenúa. Lo que acontecía podía perdurar en el tiempo y causar un daño real; no porque fuese la manifestación final de un poder alcanzado, sino porque estaba cargado con su propia capacidad de retracción, esencialmente acechado por la posibilidad de su limitada duración.

Los estudios de los afectos señalaron, a través de Deleuze, el surgimiento de una ontología diferencial - la prolífica generatividad de la diferencia y la relacionalidad que acontece en un campo de posibilidades. Lo actual se convierte en una multiplicidad de posibilidades activas en las fuerzas movilizadas por las cosas. Los estudios del afecto, junto con otras corrientes de pensamiento contemporáneo que ahora impactan la antropología (tales como el nuevo materialismo, la ontología orientada a los objetos, el debate sobre las naturculturas, las teorías multi-especie, los estudios sociales de la ciencia, las nuevas ecologías y los estudios sobre las infraestructuras materiales, y también las sociales y las afectivas, el análisis de los ritmos o las energías, y las nuevas aproximaciones radicales a la performatividad de la raza o del poder del Estado) facilitaron que esta regresara a la antigua discusión sobre cómo abrir las convenciones propias al lenguaje académico para que en ellas hubiese cabida a la generatividad y la volatilidad intrínsecas a la vida. Esto en la capacidad de la vida de cambiar o endurecerse en forma de paz o violencia, placer y dolor, colectividades y caos.

Los estudios de los afectos hacen parte de una búsqueda renovada por encontrar nuevos modos de hacer teoría y critica etnográfica, que tienen la intención de desvincularse de aquellas conversaciones académicas que toman distancia, son puramente evaluativas, y están basadas en la estabilidad de los términos y conceptos con que se trabaja. Aquí, en cambio, se trata de encontrar nuevas formas de describir lo que precede y excede a lo categórico, en el laborioso proceso de vivir en los presentes de la historia. Así, un concepto se convierte en una cosa que se despliega en una situación, a fin de intentar registrar algo acerca de las cualidades pre-personales y performáticas de la vida colectiva. Como la experiencia misma, la perspectiva etnográfica que está atenta a lo afectivo se apoya en sus objetos descriptivos con la mirada fija en cómo estos se solidifican y se convierten en algo reconocible; eso es, en algo cargado con propósitos inflexibles, susceptibles de ser desviados, o meramente transitorios. Estos movimientos han sido teorizados con solidez: Erin Manning y Brian Massumi (2014) lo llaman "pensamiento en acción" -un seguimiento detenido pero difuso a las líneas de posibilidad que, aun estando parcialmente desdobladas, son palpables. También es lo que Isabelle Stengers denomina "pragmatismo empírico" (2009). En este contexto, y siguiendo a Latour, es posible definir las articulaciones de lo empírico como un complejo proceso compositivo. O, en términos de Donna Haraway, las 
entidades y las especies son vistas como el compost de mundos naturculturales emergentes. Hay un pálpito de vida en lo material. En lo que Karen Barad (2003:817) Ilama el "proceso materializante", las cosas se hacen relevantes en tanto materialidades activas. Y la vida se hace polívoca a través del realismo de las superficies energéticas, las cualidades, y los rezagos que no tienen cabida en ejercicio de reducción y parafraseo propio a las practicas fenomenológicas y representacionales (ver Harman, 2012; 2014). Las categorías son, en el mejor de los casos, una forma oblicua de acceder a la generatividad de las singularidades en la medida en que estas acaecen (véase Harman 2008).

Los estudios de los afectos son, en este sentido, uno de los enfoques contemporáneos que nos confrontan con la posibilidad de eludir el dualismo sin salida propio a las ciencias sociales modernas y su ímpetu humanista, así como a su infortunado hábito de reprocharle todo al mundo-como si el sentido de ser y de pensar fuese, solamente, descubrir algún tipo de mentira. Como Eve Sedgwick (1997) sostiene, lo que a menudo se le llama pensamiento crítico y que se ofrece como una teoría sólida que labra su camino a través del uso de lógicas independientes a los datos analizados, no es más que una aproximación paranoica al mundo. El tan repetitivo punto de partida de este tipo de pensamiento es la convicción de que hay algo mal con la otra gente, y por lo tanto se sueña con encontrar algún mal en la forma en que el Estado ha fijado su poder o sus fantasías normativas -como si esto fuese el único problema. A diferencia de esta ceñida y desmagnetizada aproximación, los estudios de los afectos proponen un método etnográfico materializante, que ralentiza su ritmo para así recolectar el sinfín de cosas que el pensamiento crítico modernista ha omitido: todos los cuerpos, el rastro que dejan las cosas en movimiento, las bromas bien sabidas, la sonoridad, cómo cualquier trayectoria de vida lucha con un desligue no intencional, cómo comienzan las cosas, cómo la gente intenta ponerle fin a las cosas, por qué el pensamiento podría convertirse en un complemento, por qué la conceptualidad puede tomar formas radicalmente diferentes, o por qué importa que la atención se ralentice para así esperar a que algo tome forma. En tanto un modo critico de afirmación, los estudios de los afectos conspiran con los legados del pensamiento feminista, los estudios étnicos críticos, los estudios culturales, y las líneas de pensamiento en la obra de Nietzsche y Bataille, de los surrealistas y los situacionistas, de Alfred Whitehead, de Spinoza y otros más.

Los estudios del afecto ayudaron a que la antropología saliera del hábito de describir sus objetos como si estos tuviesen una forma fija. Atendiendo al desenvolvimiento de las cosas, la etnografía vuelve al compromiso de pensar desde el campo. Sin embargo, en esta ocasión el campo es definido como una serie de provocaciones y problemáticas que no podían ser resumidas en una descripción literalizada y que, por lo tanto, no desaparecían fácilmente -debido al hechizo de algún cortocircuito analítico- bajo el manto de algún paradigma extrañamente idealista que imaginaba las cosas en el mundo como un reflejo directo de sus propias distinciones categóricas. La atención analítica a las formas y las fuerzas de los cuerpos en movimiento y los eventos, invita a experimentar con la descripción y la 
conceptualización. En vez de fijar conceptos dentro de tradiciones académicas y luego imponerlos sobre lo real, podríamos pensar en el concepto como un modo de pensamiento que acontece en lo que Isabelle Stengers (Stengers, Massumi \& Manning, 2009) llama el "mezzo" o el estado de estar en medio de apegos y amenazas, de lo que queda y lo que brota. Este es un método que intenta estremecerse en sintonía con el movimiento de las cosas; de la ausencia a la presencia. Un método que hace demandas sobre los imaginarios viscerales así como del registro de lo sensorial.

Como un método etnográfico, este sería un modo de pensamiento que trataría de describir las cosas mismas que lo impulsan. Sus objetos serían sus sujetos. En vez de asumir el significado de las nociones de agencia, sujeto, objeto, cuerpos e intenciones; este intentaría describir detenidamente un mundo bajo presión, la manera en que un momento puede descender como una cortina, la forma en que un mundo se elabora de forma prolífica, ajustándose a los caminos, posándose en las personas y las prácticas. En el estado presente de emergencia y precariedad, puntos de contacto estético-material-social-político pueden emerger como presencias parpadeantes -un destello de color. O podrían surgir, espontáneamente, como esquirlas que afectan el cuerpo. La etnografía de este tipo de cosas tiene que ser ágil y paciente, saltar a un evento inesperado pero también esperar a que algunas cosas se junten. El realismo etnográfico que se busca no es plano ni incontrovertible sino que es el resultado de un proceso alquímico, viajando en circuitos de impacto y reacción. Algunas cosas ocurren en este mundo. El análisis se entrena a sí mismo, esforzándose a describir las iteraciones, duraciones, y el ocurrir de las variaciones del Ser.

\section{Referencias}

Barad, K. (2003). Posthumanist performativity: Toward an understanding of how matter comes to matter. Signs: Journal of women in culture and society, 28(3), 801-831.

Haraway, D. (2015). Anthropocene, capitalocene, plantationocene, chthulucene: Making kin. Environmental Humanities, 6(1), 159-165.

Harman, G. (2008). DeLanda's ontology: assemblage and realism. Continental Philosophy Review, 41(3), 367-383.

Harman, G. (2011). Realism without materialism. SubStance, 40(2), 52-72.

Harman, G. (2012). Weird realism: Lovecraft and philosophy. John Hunt Publishing.

Manning, E. (2009). Relationscapes: Movement, art, philosophy. MIT Press.

Manning, E., \& Massumi, B. (2014). Thought in the Act: Passages in the Ecology of Experience. University of Minnesota Press.

Pine, J. (2012). The art of making do in Naples. Minneapolis: University of Minnesota Press.

Pine, J. (2016). Last Chance Incorporated. Cultural Anthropology, 31(2), 297-318.

Sedgwick, E. K. (1997). Paranoid reading and reparative reading, or, You're so paranoid, you probably think this introduction is about you, in E. Kosofsky Sedgwick ed. Novel Gazing: Queer Readings in Fiction. Duke University Press, 1-40.

Stengers, I., Massumi, B., \& Manning, E. (2009). History through the Middle: Between Macro and Mesopolitics. An interview with Isabelle Stengers. 